\title{
Urgences pédiatriques
}

\section{Note de lecture : Urgences pédiatriques. Du prématuré à l'adolescent. P. Labrune, D. Oriot, B. Labrune et G. Huault. Édition Boeck et Estem, Paris, 2010, 2344 pages}

\section{Pediatric emergencies}

\section{P. Ray}

Reçu le 21 novembre 2011 ; accepté le 9 janvier 2012

(C) SFMU et Springer-Verlag France 2012

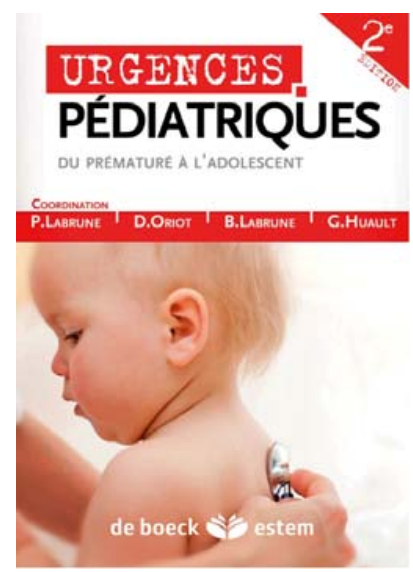

La deuxième édition du traité Urgences pédiatriques vient d'être publiée. En s'entourant de collaborateurs nationaux de renom, les éditeurs pédiatres ont réussi la gageure de réaliser un ouvrage de référence sur toutes les urgences pédiatriques, du nourrisson à l'adolescent (adolescent souvent oublié en pédiatrie d'urgence).
Multidisciplinaire et synthétique (plus de 300 figures et 420 tableaux sont présentés), ce traité associe la description de la prise en charge à partir des symptômes cliniques et description plus formelle des pathologies d'urgences les plus courantes. De plus, une grande part est laissée à la présentation synthétique des examens complémentaires et gestes d'urgences les plus utiles. Enfin, un chapitre détaillé sur la prescription médicamenteuse chez l'enfant vient compléter cet ouvrage indispensable pour ceux et celles qui s'occupent d'enfants en urgence. Seul inconvénient de cet ouvrage, il ne tient pas dans la poche de la blouse!

P. Ray $(\bowtie)$

Service d'accueil des urgences,

Groupe hospitalier Tenon-Saint Antoine,

4, rue de la Chine, F-75020 Paris,

Université Pierre et Marie Curie-Paris 6, France

e-mail : patrick.ray@psl.aphp.fr 